\title{
Near Field Properties and Plasmonic Effects of a Periodic Bowtie Antenna Array Embedded in a Substrate with Different Depths
}

\author{
Yuan-Fong Chou Chau, San-Cai Jheng, Wayne Yang, and Ci-Yao Jheng
}

\begin{abstract}
We numerically investigate the near field properties and plasmonic effects of a periodic bowtie antenna array which is embedded in a substrate with different depths by means of finite element method with three-dimensional calculations. The electromagnetic modes obtained from the embedding cases are quite different from that of air background case of the same size, resulting in an intensity enhancement and a redshift phenomenon. We find that the embedded depth of the bowtie antenna in a silica substrate is an important parameter which can influence the field enhancement and the position of peak resonant wavelengths. The near-field intensity becomes less intense and the spectrum of peak resonances red-shifted as the embedded depth is increased.
\end{abstract}

Index Terms-Bowtie antenna, finite element method, plasmonic effects, red-shifted.

\section{INTRODUCTION}

The electromagnetic of plasmonic waves are excitations propagating at the interface between a metal and a dielectric medium, evanescently confined in the normal direction [1]-[3]. An important field pattern of the excitation of plasmonic waves is significant enhancement of the incident electric fields near the surface of the metallic regions by several orders of magnitude [4]. In recent years, plasmonic antennas [5]-[8] have attracted of great interest by researches owing to their ability to support the localized surface plasmon resonance (SPR) and provide the enhanced and confined electromagnetic fields. Various applications of plasmonics antennas have been proposed and demonstrated [9]-[12]. Besides, their applications can be used in biological sensing and imaging. These plasmonic antennas can be used to manipulate nanoparticles that are attracted by the high intense fields generated in the gap between the metallic regions. The optical properties of different types of plasmonic antennas have been discussed over the last few years [see, for example [5], [9], [13]-[15]. In our previous paper, we first proposed a bowtie antenna with a dielectric hole enclosed by a silver nanoshell to tune the peak resonant wavelength $\left(\lambda_{\text {res }}\right)$ of the antenna [5], [9]. The peak resonance

Manuscript received January 3, 2013; revised March 25, 2013. This work was supported in part by the National Science Council of the Republic of China (Taiwan) under Contracts NSC 99-2112-M-231-001-MY3, NSC 101-3113-P-002-021-

The authors are with the Chien Hsin University of Science and Technology, Zhongli, Taiwan 320, R.O.C. (e-mail: yfc01@ uch.edu.tw, rock1989123@yahoo.com.tw, wayen@uch. edu.tw, m10011003@uch. edu.tw). wavelength of a nanoshell bowtie antenna can be tuned from $\lambda_{\text {res }}=635 \mathrm{~nm}$ to $\lambda_{\text {res }}=732 \mathrm{~nm}$ and a $97 \mathrm{~nm}$ redshift is obtained by varying the dielectric hole of $\varepsilon=5$ filled inside the nanoshell bowtie antenna. Our other papers also show that the peak resonance wavelength of a nanoshell antenna may be tuned by the geometrical parameters [10], [11]. The drawback of our proposed design in [9] is that it would require the complex procedures to fabricate it [5]. Thus, in the view point of fabrication and cost, the solid bowtie antenna is the best choice in this issue.

When a solid bowtie antenna is embedded in an air background and is illuminated with the incident wave of preferential wavelengths, the antenna will be strongly polarized and the incident fields can be enhanced by several orders of magnitude in the antenna gap [4], [16]. However, the plasmonic effects of a periodic antenna array embedded in a substrate have never been investigated. In this paper, we numerically investigate a periodic array of plasmonic bowtie antenna which is composed of a pair of identical triangular gold. We will examine the influence of its embedded depths in a silica substrate on the antenna resonance conditions compared to the air background case of counterpart. Numerical simulations are performed by using three-dimensional (3-D) finite element method (FEM). We examine the near field properties on the antenna resonance conditions, such as peak resonance wavelengths, gap enhancements, propagation properties, charge densities and electrical filed stream lines at peak wavelengths of interest. In addition, the characteristics of transmittance spectral of the proposed periodic antenna array corresponding to their bonding mode and anti-bonding mode are investigated as well.

The structure of a unit cell of the periodic bowtie antenna array investigated here can be characterized by five parameters $(a, b, c, d, e)$ as shown in Fig. 1: the gap width, $d$; the antenna length along x-axis, $a$; the antenna width along $\mathrm{y}$-axis, $b$; and the antenna thickness, $c$. It should also be noted that the area within the gap has an eventful impact on the optical performance of antenna. Limited by the uncertainty of conventional nanofabrication, it is often difficult to fabricate antenna arrays with a reproducible gap size below $20 \mathrm{~nm}$ [6], therefore a gap width of $d=30 \mathrm{~nm}$ is chosen throughout this paper. The area of gap region $d \times e=30 \mathrm{~nm} \times 30 \mathrm{~nm}$ is chosen for this study. In addition, the gold film thickness is kept constant of $c=35 \mathrm{~nm}$. The dielectric constants of gold were obtained from literature [17]. An antenna flare angle of $90^{\circ}$ is used, such that $a=b$. In each simulation, a unit cell of bowtie antenna is embedded in the air background or embedded with 
a depth of $v \mathrm{~nm}$ in a fused silica $\left(n_{s}=1.5\right)$ substrate, where the interface between air and silica substrate is set to be zero (i.e., the origin of the coordinate system is placed at the center of gold/silica interface), while positive $v$ and minus $v$ denote the distance between the top surface of antenna and the interface (top surface of silica). The amplitude of the incident light is set to be $1 \mathrm{~V} / \mathrm{m}$ throughout this paper. We define a term "gap enhancement" which means the near-field intensity measured at the gap region. In our simulations, we use 3-D FEM, with triangular high-order edge elements. On the surface of outer simulation region, the case of a unit cell of the periodic bowtie antenna array is enclosed by the anisotropic perfectly matched layers (PML) in order to avoid the reflective fields from the boundary of the computational domain. For simulating the periodic bowtie antenna arrays, we set periodic boundary condition (PBC) along the side walls and PML condition on the top and bottom surfaces in order to save the computer resource and simulation time.

\section{Simulation Method, Results AND DisSCUSSION}

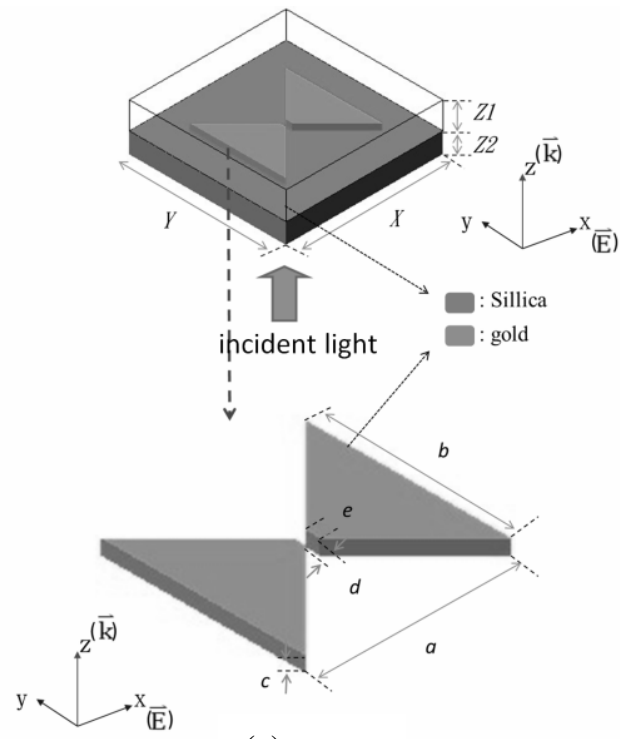

(a)

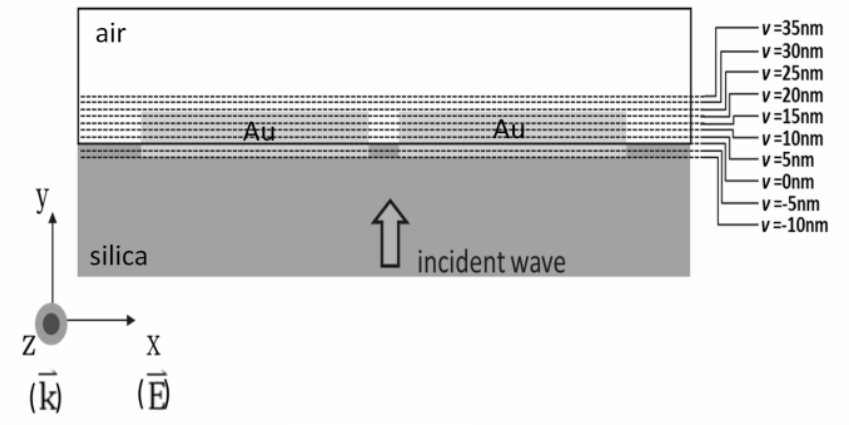

(b)

Fig. 1. Schematic of the simulation model. (a) The structure of a bowtie antenna with five structural parameters $(\mathrm{a}, \mathrm{b}, \mathrm{c}, \mathrm{d}, \mathrm{e})$, the area of gap region, $d \times$ $e=30 \mathrm{~nm} \times 30 \mathrm{~nm}$; the gold film thickness, $\mathrm{c}=35 \mathrm{~nm}$; antenna flare angle, $90^{\circ}$, antenna length and width, $a=b=475 \mathrm{~nm}$; a silica substrate, $n s=1.5$. (b) Side view of an antenna embedded in a silica substrate. The positive $\mathrm{v}$ denotes the distance between the antenna and silica, while minus $\mathrm{v}$ denoting the embedded depth of the antenna. The dashed lines exactly touch the top surface of the antenna.
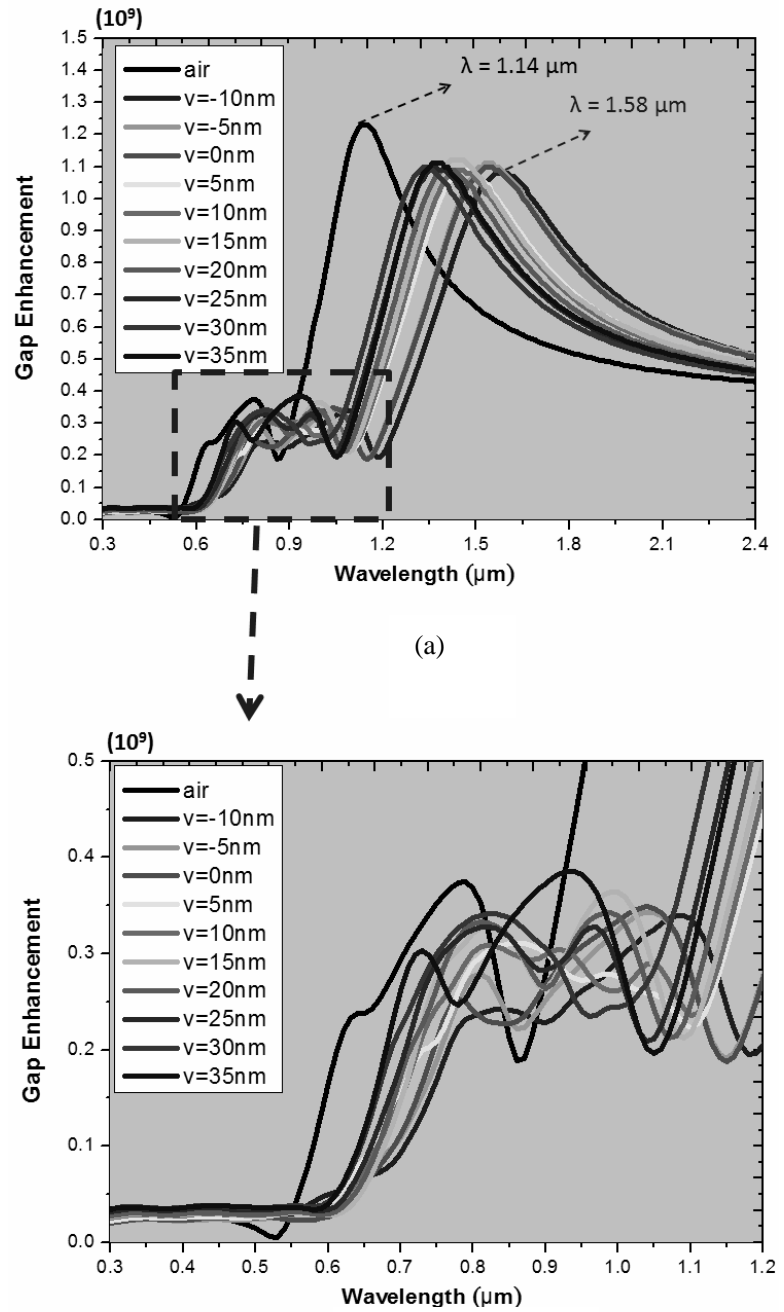

(b)

Fig. 2 (a) Difference of the gap enhancement vs. wavelengths obtained from air background case (black line) and embedding cases with varying the embedded depths of antenna in a silica substrate ranging in $-10<v<35 \mathrm{~nm}$. (b) The enlarged diagram of Fig. 2 (a) which incident wavelengths ranging in $0.3-1.2 \mu \mathrm{m}$.

First, we investigate the difference of the gap enhancement vs. wavelengths obtained from air background case and embedding cases of antennas with varying the embedded depths in a silica substrate ranging in $-10<v<35 \mathrm{~nm}$ as shown in Figs. 2 (a) and (b). In our calculations, the $x$-polarized electromagnetic incident wave launches from the bottom face of the substrate and propagates along the positive $z$ direction, which is used to excite the local field surrounding the surface of bowtie antenna (see the inset of Fig. 1). The geometry parameters are used as follows: $d=e=30 \mathrm{~nm}, a=b=500 \mathrm{~nm}$ and $c=35 \mathrm{~nm}$, respectively. The SPs significantly increase the field enhancement within the antenna gap and antenna surface. As shown in Fig. 2 (a) that at least two distinct resonance peaks corresponding to the SPR mode can be found. Fig. 2 (b) also shows the enlarged diagram of Fig. 2 (a) which wavelengths are in the range of $0.3-1.2 \mu \mathrm{m}$. It is evident in Fig. 2 that the peak resonance wavelength of the bowtie antenna is always at a longer wavelength when the embedded depth $v$ is increased and peak resonance wavelength $\left(\lambda_{\text {res }}\right)$ redshifts to longer wavelengths and the gap enhancement is decreased owing to absorption effects of the dielectric substrate (silica). The second $\lambda_{\text {res }}$ shifts from 1.14 
$\mu \mathrm{m}$ (for air background case) to $1.68 \mu \mathrm{m}$ (for case $\nu=-10 \mathrm{~nm}$ ) and a distinct redshift of $440 \mathrm{~nm}$ is obtained. This phenomenon can be explained by the polarization effects of the silica substrate and by the plasmonic effects arising from the interaction between the metal antenna and the applied incident field, which will be discussed in Fig. 4. Note that the gap enhancements of the embedding cases revealed in Fig. 2 (b) are achieved a value above $2 \times 10^{8}$ as the incident wavelengths larger than $0.775 \mu \mathrm{m}$.
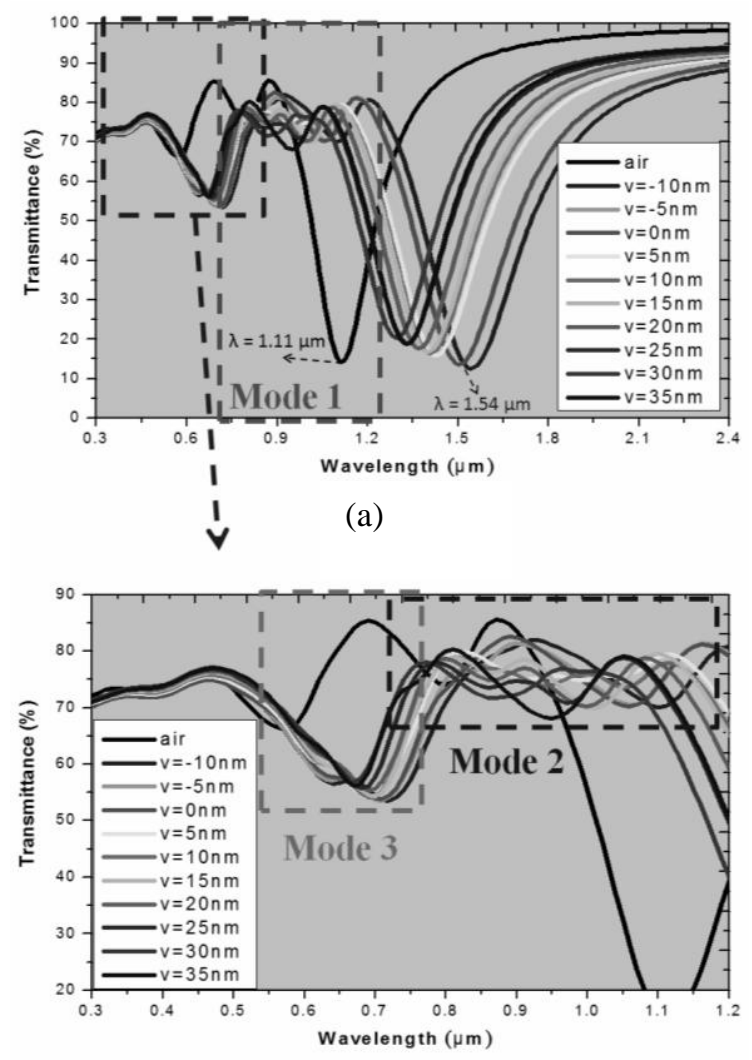

(b)

Fig. 3 (a) Difference of the transmittance vs. incident wavelengths obtained from air background case (black line) and embedding cases with varying the embedded depths of antenna in a silica substrate ranging in $-10<v<35 \mathrm{~nm}$. (b) The enlarged diagram of Fig. 3 (a) which incident wavelengths ranging in $0.3-1.2 \mu \mathrm{m}$.

Plasmonic antenna consists of two strongly coupled nanometal particles which can serve as a model system to study the impact of mode selectivity. Fig. 3 shows the difference of the transmittance vs. incident wavelengths obtained from air background case and embedding cases with varying the embedded depths of antenna in a silica substrate ranging in $-10<v<35 \mathrm{~nm}$, whereas the enlarged diagram of Fig. 3 (a) which incident wavelengths ranging in $0.3-1.2 \mu \mathrm{m}$ is displayed in Fig. 3 (b). It can be seen from Fig. 3 (a) that three different transmittance modes can be found, i.e., mode 1, 2, 3 (we name them as mode 1, 2 and 3 hereafter), as indicated in the enclosed dashed-lines of Fig. 3 (a) and 3 (b). The corresponding transmittance modes and their charge distributions are also displayed in Figs. 4 (a)-(c), respectively A quite different field patterns and charge distributions can be found, showing bonding (see mode 1, Fig. 3 (c)) and anti-bonding (see mode 2, 3, Fig. 3 (a), (b)) mode patterns. Comparison of the results obtained from Figs. 3(a) with 3(b), the transmittance dips in mode 1 are much deeper than those of mode 2 and 3 , and redshifts towards longer wavelengths as the decreasing value of $v$. These results in the splitting of the plasmon mode into two resonance modes, which are the low energy "bonding" mode (mode 1) and the high energy "anti-bounding" mode (mode 2 and 3 ). Because of coupling effects between two gold nanoparticles of the bowtie antenna, we find that the electric field is enhanced and forms a hot spot in the gap for modes 1-3 (see the bottom part of Figs. 4 (a)-(c)).

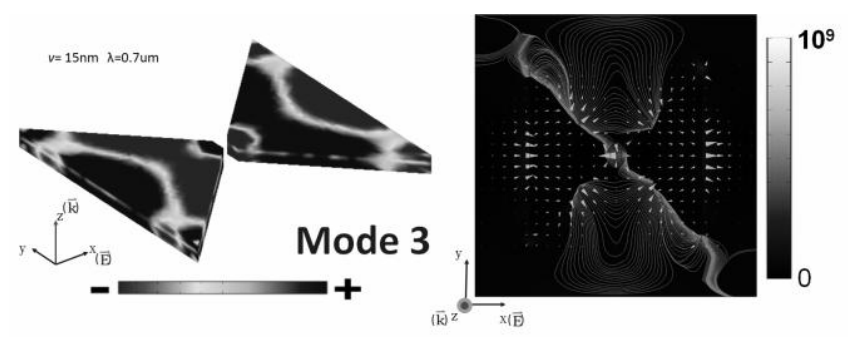

(a)

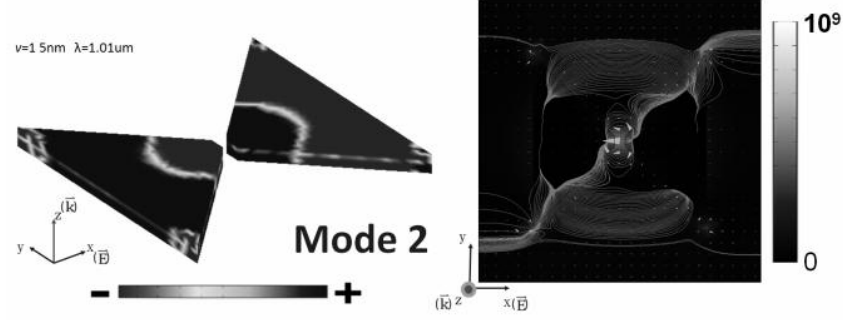

(b)

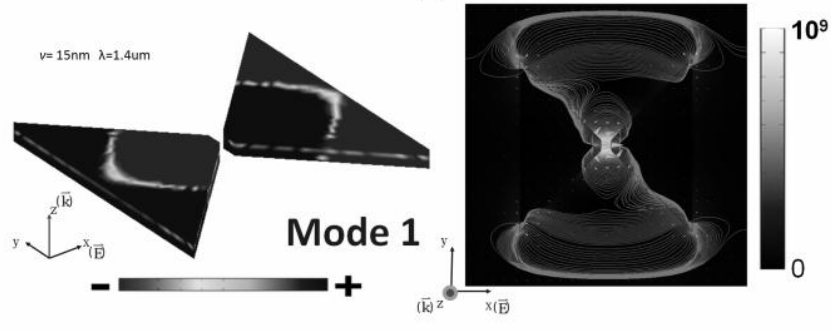

(c)

Fig. 4. Corresponding charge distributions and their transmittance modes of mode 1-3. (a) Mode 3, (b) Mode 2, (c) Mode 1, respectively.

Regarding the direction of electric field propagation (white arrows in the bottom part of Figs. 4 (a)-(c)), the schematic charge density of the mode 1-3 are also depicted in the upper part of Figs. 4 (a)-(c), respectively. In mode 1 case, the charge density (see Fig. 4 (c)) on the surfaces of the antenna are divided into two parts in the form of dipole arrangement and the charge intensity are stronger than that of mode 2 and 3 cases, showing strong dipole-like charge distributions and resulting in bonding mode resonance. Turning to the mode 2 and 3 cases (see Fig. 4 (a) and (b)), the charge densities distribute more than two areas and exhibit weaker dipole-like than that of mode 1, leading to an anti-bonding mode resonance. The mechanism on above-mentioned phenomena can be attributed to the symmetries of the positive-negative charge distributions and their distribution profile for gold bowtie antenna arrays. The dipole moments on the antenna surfaces of outline are arranged. A stronger coupling will lead to a new scheme of polarization and result in a thin metallic layer, which could be described as symmetric and asymmetric modes. 

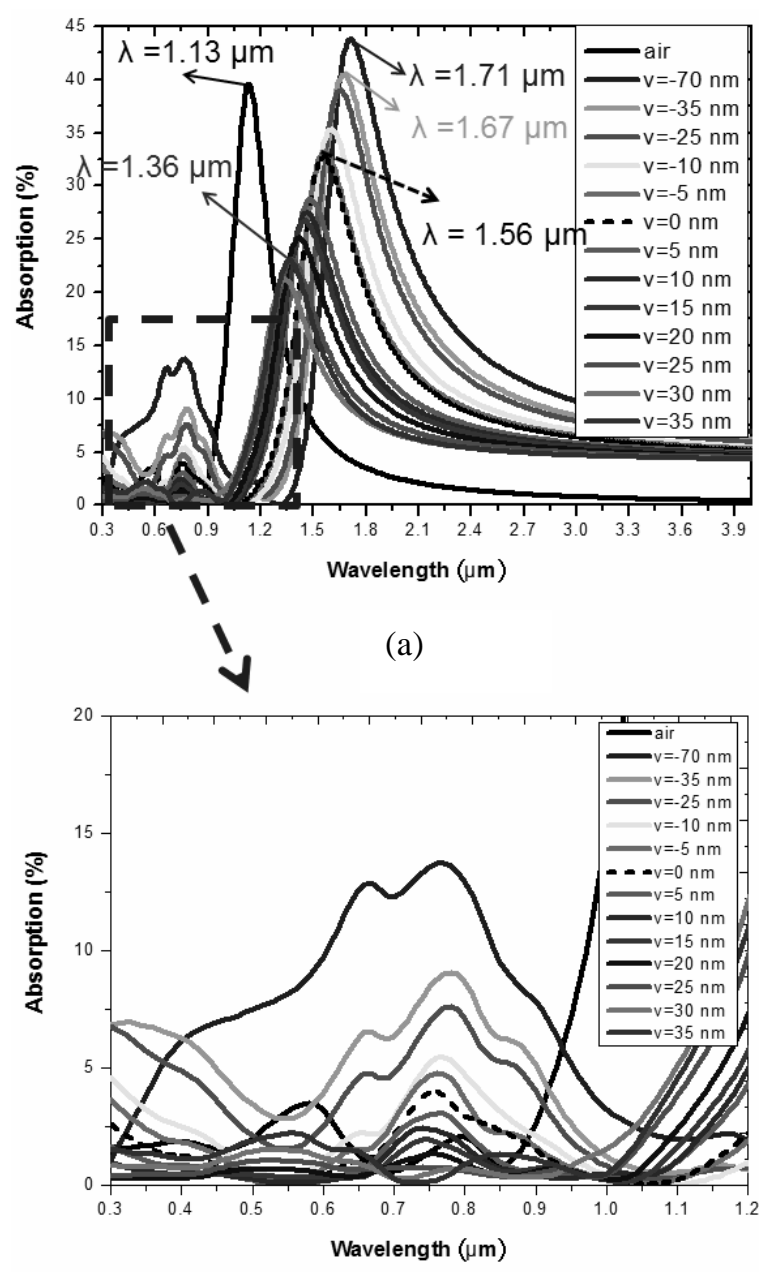

(b)

Fig. 5 (a) Difference of the transmittance vs. incident wavelengths obtained from air background case (black line) and embedding cases with varying the embedded depths of antenna in a silica substrate ranging in $-10<v<35 \mathrm{~nm}$. (b) The enlarged diagram of Fig. 3 (a) which incident wavelengths ranging in $0.3-1.2 \mu \mathrm{m}$.

Fig. 5 (a) also shows the difference of the absorption vs. incident wavelengths obtained from air background case (black line) and embedding cases with varying the embedded depths of antenna in a silica substrate ranging in $-10<v<35$ $\mathrm{nm}$. The enlarged diagram of Fig. 5 (a) which incident wavelengths ranging in $0.3-1.2 \mu \mathrm{m}$ is also shown in Fig. 5 (b). It is evident from the figure as shown in Fig. 5 (a), the absorption peaks can be shifted from $1.13 \mu \mathrm{m}$ (air background case) to $1.71 \mu \mathrm{m}$ (embedding depth of $v=-70 \mathrm{~nm}$ ) and a significant redshift of $580 \mathrm{~nm}$ is obtained. Note that the position of absorption resonance peak wavelength is a little different from that of transmittance resonance peak wavelength owing to the reflection effect is considered in our simulations. From Fig. 5, a useful application can be applied in solar cell, which absorption efficiency can be enhanced and the operation wavelength can be tuned when the embedding depth is increased.

When the antenna is aligned along a particular direction, the symmetry of the system is broken, and proper modes can be found for light polarized parallel to the chain axis of the periodic bowtie antenna. Independently of the direction of the applied field, the spectrum is always red-shifted as the antennas approaching and embedding the substrate [18]. This can be explained using the real and image charge model as depicted in Fig. 6 (model of the induced local field for an applied field parallel to the antenna), Figs. 6 (a)-(c) represent the case of $v=-10 \mathrm{~nm}$ (i.e., the distance between top surface of antenna and the interface) corresponding to mode 1, 2 and 3, respectively. It can be clearly seen in Fig. 6 that the induced local electric field $\mathbf{E}_{\text {ind }}$ is opposite to the external field $\mathbf{E}_{\text {ext }}$. When the external field is applied parallel to the interface, it polarizes the particle in the opposite direction to the substrate polarization, thus the induced local field acting on the particle is also along the applied field, such that, the induced local field $\mathbf{E}_{\text {ind }}$ is in the opposite direction as the applied field, see Fig. 6 (a)-(c), and against the restoring forces, thus increasing the wavelength of the SPRs. $(\overrightarrow{\mathrm{k}})$

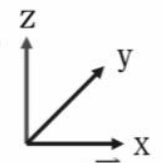

$(\overrightarrow{\mathrm{E}})$

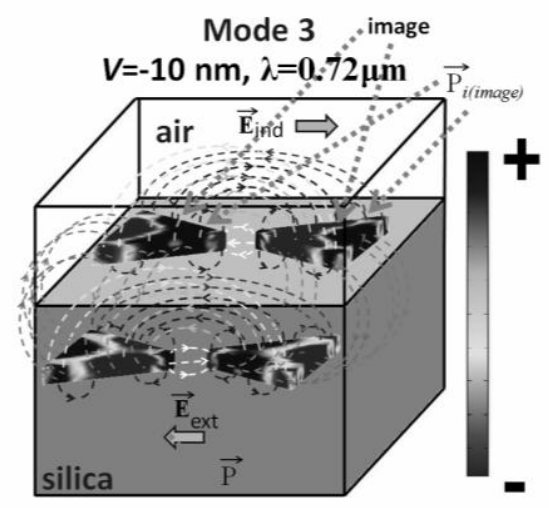

(a)
Mode 2 , image

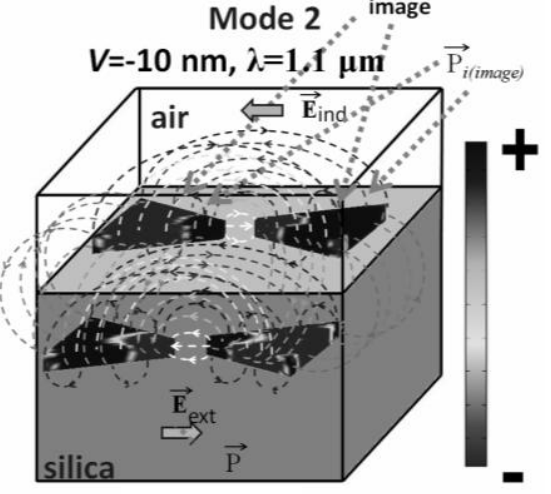

(b)

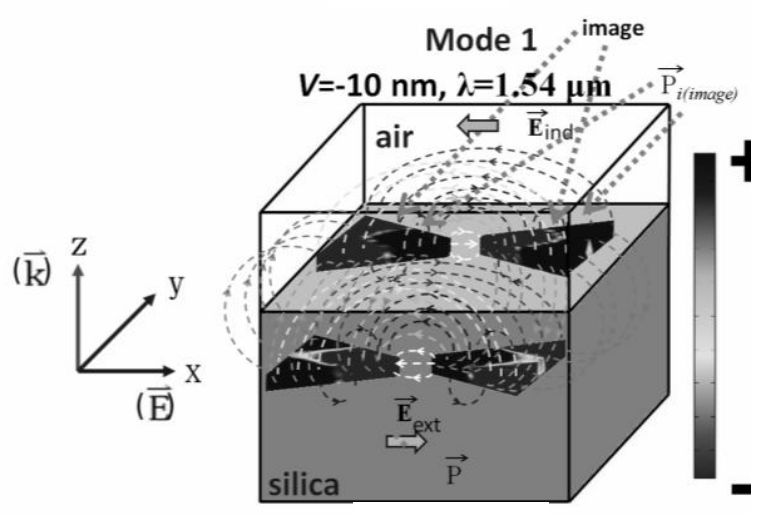

(c)

Fig. 6 Model of the induced local field for an applied field parallel to the plasmonic bowtie antenna for the selected case of $v=-10 \mathrm{~nm}$. (a)-(c) represent the bowtie antenna with embedded depth of $\mathrm{v}=-10 \mathrm{~nm}$ corresponding to their peak wavelengths ( $\lambda$ res), respectively. (a) $\lambda$ res $=0.72 \mu \mathrm{m}$, (b) $\lambda$ res $=1.1 \mu \mathrm{m}$, (c) $\lambda$ res $=1.54 \mu \mathrm{m}$. 


\section{CONCLUSION}

In summary, we have numerically investigated the near field properties and plasmonic effects of a periodic bowtie antenna array embedded in a substrate with different depths by using FEM with three-dimensional calculations. The gap enhancement, transmittance, absorption, charge distribution and the real and image charge model have been investigated and discussed in our 3-D calculations. Results show that the investigated antenna embedding with different depths in a silica substrate, offering more compact dimensions and a broader spectral while maintaining a constant antenna size. It is anticipated that these results will inspire further developments in plasmonic antennas with complex geometries and will have future applications in near- to mid-infrared spectroscopy and sensing.

\section{ACKNOWLEDGMENT}

The authors acknowledge the financial support from the National Science Council of the Republic of China (Taiwan) under Contracts NSC 99-2112-M-231-001-MY3, NSC 101-3113-P-002-021-.

\section{REFERENCES}

[1] H. T. Hattori, Z. Li, D. Liu, I. D. Rukhlenko, and M. Premaratne, "Coupling of light from microdisk lasers into plasmonic nano-antennas,” Opt. Express, vol. 17, pp. 20878-20884, 2009.

[2] J. Kottmann, O. Martin, D. Smith, and S. Schultz, "Spectral response of plasmon resonant nanoparticles with a non-regular shape," Opt. Express, vol. 6, pp. 213-219, 2000.

[3] H. Wang, D. W. Brandl, F. Le, P. Nordlander, and N. J. Halas, "Nanorice: A hybrid plasmonic nanostructure," Nano Lett., vol. 6, pp. 827-832, 2006.

[4] S. A. Maier, P. G. Kik, H. A. Atwater, S. Meltzer, E. Harel, B. E. Koel, and A. A. G. Requicha, "Local detection of electromagnetic energy transport below the diffraction limit in metal nanoparticle plasmon waveguides," Nat. Mater., vol. 2, no. 4, pp. 229-232, 2003.

[5] S. Sederberg and A. Y. Elezzabi, "Nanoscale plasmonic outline bowtie antenna operating in the mid-infrared," Opt. Express, vol. 19, pp. 15532-15537, 2011.

[6] J.-S. Huang et al., "Mode Imaging and Selection in Strongly Coupled Nanoantennas," Nano Lett., vol. 10, pp. 2105-2110, 2010.

[7] S. Aksu, A. A. Yanik, R. Adato, A. Artar, M. Huang, and H. Altug, "High-throughput Nanofabrication of Plasmonic Infrared Antenna Arrays for Vibrational Nanospectroscopy," Nano. Lett., vol. 10, pp. 2511-2518, 2010.

[8] J.-S. Huang, J. Kern, P. Geisler, P. Weinmann, M. Kamp, A. Forchel, P. Biagioni, and B. Hecht, "Mode Imaging and Selection in Strongly Coupled Nanoantennas," Nano Lett., vol. 10, pp. 2105-2110, 2010.

[9] Y. F. Chau, H. H. Yeh, and D. P. Tsai, "A new type of optical antenna: plasmonics nanoshell bowtie antenna with dielectric hole," $J$. Electromagn. Waves Appl., vol. 24, pp. 1621-1632, 2010.

[10] Y. F. Chau, H. H. Yeh, and D. P. Tsai, "Surface plasmon resonances effects on different patterns of solid-silver and silver-shell nanocylindrical pairs," J. Electromagn. Waves Appl., vol. 24, pp. 1005-1014, 2010.

[11] Y. F. Chau and H. H. Yeh, "A comparative study of solid-silver and silver-shell nanodimers on surface Plasmon resonances," J. Nanopart. Res., vol. 13, pp. 637-644, 2011.

[12] L. Wang, L. Cai, J. Zhang, W. Bai, H. Hu, and G. Song, "Design of plasmonic bowtie nanoring array with high sensitivity and reproducibility for surface-enhanced Raman scattering spectroscopy," J. Raman Spectrosc, vol. 42, pp. 1263-1266, 2011.

[13] G. Leveque and O. J. F. Martin, "Tunable composite nanoparticle for plasmonics," Opt. Lett., vol. 31, pp. 2750-2752, 2006.

[14] O. L. Muskens and J. A. S.-G. V. Giannini, and J. G'omez Rivas, "Optical scattering resonances of single and coupled dimer plasmonic nanoantennas," Opt. Express, vol. 15, pp. 17736-17746, 2008.

[15] A. Alu and N. Engheta, "Tuning the scattering response of optical nanoantennas with nanocircuit loads," Nature Photonics, vol. 2, pp. 307-309, 2008.
[16] F. Neubrech, A. Pucci, T. W. Cornelius, S. Karim, A. Garcia-Etxarri, and J. Aizpurua, "Resonant Plasmonic and Vibrational Coupling in Tailored Antenna for Infrared Detection," Phys. Rev. Lett., vol. 101, pp. 157403, 2008.

[17] A. D. Rakic, A. B. Djurisic, J. M. Elazar, and M. L. Majewski, "Optical properties of metallic films for vertical-cavity optoelectronic devices,' Appl. Opt., vol. 37, pp. 5271-5283, 1998.

[18] C. Noguez, "Surface Plasmons on Metal Nanoparticles: The Influence of Shape and Physical Environment," J Phys Chem C 111, pp. 3806-3819, 2007.

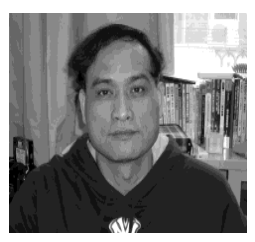

Yuan-Fong Chau was born on August 23, 1963, in Hualian, Taiwan. He received his M.S. of Electronic Engineering in Chung Chein Institude of Techonology, Da-Shi, Taiwan from 1983-1985. He also received his second M.S. of Physics in National Taiwan University, Taipei city, Taiwan from 1983-1985. He received his Ph.D degree in Department of Electrophysics from Chiao Tung University, Taiwan from 2001-2004.

From 1985 to 2004 he was an officer of Taiwan Army. He was an Assistant Professor at department of electrical Engineering, Lee Min institute of technology, New Taipei city, Taiwan from 2004 to 2005. From 2005 to 2008 he was an Assistant Professor in department of electrical engineering, Ching Yun university, Zhongli, Taiwan. From 2008 to 2012 he was an Associate Professor in department of electrical engineering, Ching Yun university, Zhongli, Taiwan. Now he is an Associate Professor in department of electrical engineering, Chien Hsin University of Science and Technology, Zhongli, Taiwan.

$\mathrm{He}$ is the author and coauthor of 55 SCI journal papers and more than 50 conference papers. He had 6 patents in Taiwan. His current research interests are nano-photonics, near-field optics, plasmonics, photonic crystals, meta-materials, bio-photonics and their applications. He was invited as an invited speaker for international conference or symposium more than 10 times. He has received a number of prestigious awards including "Best poster paper awarded, The international conference on nanophotonics 2010, Tsukuba, Japen, 2010," "Silver Medal, The international Innovative Invention Poster Competition for Biotechnology, Taiwan, 2010," and " Award for publication of Taiwan aboriginal people, From 2008 to 2012'.

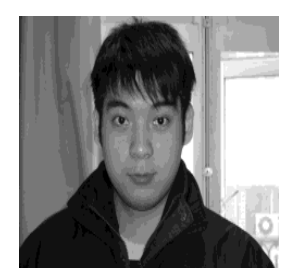

San-Cai Jheng was born in New Taipei city, Taiwan on January 23, 1989. He received his M.S. of Electronic Engineering in Chien Hsin University of Science and Technology, Zhongli, Taiwan from 2011-2013.

$\mathrm{He}$ is the author and coauthor of $3 \mathrm{SCI}$ journal papers and more than 10 conference papers.

His current research interests are nano-photonics, near-field optics, plasmonics, photonic crystals, meta-materials, bio-photonics and their applications.

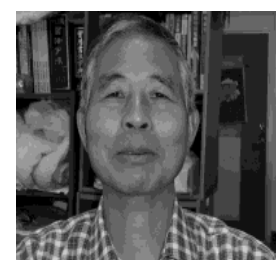

Wayne Yang was born in Taoyuan city, Taiwan on March 26, 1954. He received his M.S. of Electrical Engineering in Tennessee tech university, Cookeville, U.S.A. from 1981-1983. He was a lecturer at department of electronic Engineering, Lee Min institute of technology, New Taipei city, Taiwan from 1979 to 1981. From 1983 to 2011 he was a lecturer in department of electronic engineering, Ching Yun university, Zhongli, Taiwan. Now he is a lecturer in department of electronic engineering, Chien Hsin University of Science and Technology, Zhongli, Taiwan.

$\mathrm{He}$ is the author and coauthor of $3 \mathrm{SCI}$ journal papers and more than 10 conference papers. His current research interests are nano-photonics, near-field optics, plasmonics, photonic crystals, meta-materials, bio-photonics and their applications.

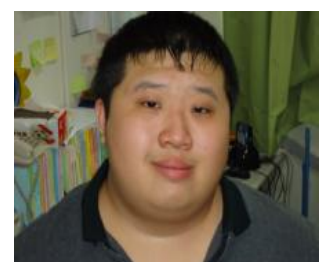

Ci-Yao Jheng was born in New Taipei city, Taiwan on December 16, 1988. He received his M.S. of Electronic Engineering in Chien Hsin University of Science and Technology, Zhongli, Taiwan from 2011-2013.

$\mathrm{He}$ is the author and coauthor of $3 \mathrm{SCI}$ journal papers and more than 10 conference papers.

His current research interests are nano-photonics, near-field optics, plasmonics, photonic crystals, meta-materials, bio-photonics and their applications. 